\title{
Population biology of Toxoplasma gondii: what's out and where did they come from
}

\author{
JP Dubey ${ }^{1 /+}$, Chunlei Su${ }^{2}$ \\ 1Animal Parasitic Diseases Laboratory, ANRI, ARS, USDA, Building 1001, Beltsville, 20705-2350 Maryland, USA 2Department of \\ Microbiology, The University of Tennessee, Knoxville, Tennessee, USA
}

Until recently, Toxoplasma gondii was considered to be clonal with little genetic variability. In this paper we summarize recent genotyping data from chickens in Brazil, and pigs, lambs and white-tailed deer in the USA, to demonstrate the high genetic diversity and geographical distribution of T. gondii. A total of $149 \mathrm{~T}$. gondii isolates from 13 geographical areas of Brazil and $182 \mathrm{~T}$. gondii isolates from pigs, 53 isolates from sheep and 15 isolates from fetal white-tailed deer from USA were genotyped using the 10 RCFP-PCR genetic markers SAG1, SAG2, SAG3, BTUB, GRA6, c22-8, c292, L358, PK1 and Apico. Genotyping of 149 T. gondii isolates from free range chickens in Brazil identified 58 genotype groups. No clonal type II lineage was found. Of the 253 isolates from animals from USA, 18 genotypes were identified, predominantly type II. These studies indicate a higher genetic diversity than previously recognized.

Key words: Toxoplasma gondii - genotyping - oocysts - tissue cysts - worldwide

Toxoplasma gondii is one of the most successful parasites. There is only one species in the genus. It infects virtually every warm-blooded vertebrates, including humans and livestock. One reason for its widespread distribution to all continents is its adaptability to be transmitted by several modes. Historically, it is a coccidian parasite and all coccidians were probably onehost parasite with fecal-oral transmission (Dubey 2007). With domestication, it has adapted to be transmitted also congenitally and by carnivorism. However, cat is essential in the life cycle of $T$. gondii. Seroepidemiological studies on isolated islands in the Pacific (Wallace et al. 1972), Australia (Munday 1972) and the USA (Dubey et al. 1997) have shown an absence of $T$. gondii on island without cats. Vaccination of cats with a live mutant strain of T. gondii (T-263) on eight pig farms in the USA reduced the transmission of $T$. gondii infection in mice and pigs (Mateus-Pinilla et al. 1999), supporting the role of the cat in natural transmission of $T$. gondii. One adaptation of the parasite is high infectivity of oocysts to herbivores and bradyzoites to cats. Cats fed a few bradyzoites can shed many oocysts and as few as one oocyst can infect a pig. Although infective dose of oocysts for humans is unknown (and there is no rush to determine it), oocyst-induced infections are considered more severe than after ingestion of tissue cysts. There is intense debate whether virulence of the parasite contributes to the severity of disease in humans (Dubey \& Jones 2008). T. gondii was considered to be clonal with little genetic variability (Howe \& Sibley 1995). However, recent studies by us and several researchers have shown that the parasite is more genetically diverse than realized earlier.

+ Corresponding author: jitender.dubey@ars.usda.gov

Received 10 October 2008

Accepted 29 October 2008
In addition, Lehmann et al. (2006) recognized intercontinental differences among $T$. gondii strains by microsatellite analysis. Here, we summarize recent genotyping data from chickens in Brazil, and pigs, lambs and whitetailed deer in the USA, to demonstrate the high genetic diversity and geographical distribution of $T$. gondii.

\section{MATERIALS AND METHODS}

Rationale - Seven years ago, we planned a worldwide study of $T$. gondii population structure (Dubey et al. 2002, Lehmann et al. 2006). Unlike previous studies that used isolates from an assortment of hosts and mostly from clinical human cases from Europe and North America, we sought to elucidate the population structure of $T$. gondii using previously unanalyzed, asymptomatic infections in a single host species. Initially, we concentrated on the free-range back yard chickens, because they feed from the ground and probably become infected by ingesting $T$. gondii oocysts from the ground, and their confinement to a house or a small farm. The plan was to obtain only a few chickens from one source so that many strains from different sources can be obtained. The plan was to bioassay all chickens, irrespective of their $T$. gondii antibody status, to avoid selection of strains for the study. All bioassays were made in one laboratory (in the laboratory of JPD) to avoid variability. Sera of chickens were tested with the modified agglutination test starting at 1:5 serum dilution. Tissues (brain, heart) of seropositive chickens were digested in pepsin and the homogenate inoculated subcutaneously into four or five mice. The day of death of each mouse was recorded. Lungs of mice that died and the brains of mice killed six weeks post-inoculation were examined for $T$. gondii and attempts were made to freeze tissues for DNA extraction. Viable parasites from one mouse from each infected chicken were stored in liquid nitrogen. After the outbreak of avian flu in south-east Asia and other countries we could not import chicken tissues from affected countries because of restrictions imposed by the 
USA authorities on international transport of chickens. Therefore, we switched to dogs and cats, and wildlife tissues as source of $T$. gondii strains and these studies are on going. In the present paper we will give example of our studies on $T$. gondii strains from chickens from Brazil and compare data on strains from two food animals (pigs and sheep) and one wild animal (white-tailed deer).

T. gondii isolates from chickens from Brazil - A total of $149 \mathrm{~T}$. gondii isolates from 13 geographical areas, including the states of Alagoas, Bahia, Ceará, Maranhão, Paraná, Pernambuco, Rio de Janeiro, Rio Grande do Norte, São Paulo, Sergipe, Rondônia, Pará and Rio Grande do Sul (Figure, Table) of Brazil were genotyped (Dubey et al. 2008c).

T. gondii isolates from domestic pigs from USA - A total of 182 ( 85 from sows from Iowa, 65 from market pigs from two farms in Massachusetts and Maryland, 29 from market pigs from slaughter house in Pennsylvania and three from retail store pork) $T$. gondii (TgPgUs1-182) were examined (Dubey et al. 2008a, Velmurugan et al. 2009).

T. gondii isolates from lambs from USA - A total of 53 isolates (TgShUs1-53) from lambs slaughtered in Maryland and Virginia were examined (Dubey et al. 2008b).

T. gondii isolates from deer from USA - A total of 15 T. gondii (TgWtdUs1-15) isolates from fetal white-tailed deer from Iowa and Minnesota were tested (Dubey et al. 2008d).

Genetic typing - For the present study, T. gondii DNA was extracted from tissues of infected mice and geno- typed using the 10 PCR-RFLP genetic markers including SAG1, SAG2, SAG3, BTUB, GRA6, c22-8, c29-2, L358, PK1 and Apico (Dubey et al. 2006, Su et al. 2006).

\section{RESULTS}

Chickens from Brazil - Genotyping of 149 T. gondii isolates from free range chickens in Brazil identified 58 genotype groups (Genotype \#1-58) (Figure, Table). Twenty-nine of the 58 multilocus genotypes identified are characterized with two or more isolates, while the other 29 genotypes have single isolate each. Only one ( TgCkBr146) of 151 isolates was clonal type I strain and five were clonal type III strains. No clonal type II lineage was found. One strain $(\mathrm{TgCkBr} 168)$ was type II at all loci, except BTUB. The four major $T$. gondii lineages (types BrI, BrII, BrIII and BrIV) previously identified from different animal hosts in Brazil (Pena et al. 2008) were also identified from chickens from much wider geographical regions in this study. In addition, the genotype group \#21 was also identified in several states in Brazil. Phylogenetic relationships of these 149 chicken isolates showed no clear clustering of genotypes with different geographical regions and the lack of type II alleles in the parasite population in Brazil. In addition, most genotypes are found only in one of the 13 states analyzed in this study, with exception of the lineages type III, BrI, BrII, BrIII, BrIV and genotype groups \#21 (with 10 isolates from six different states in Brazil).

Pigs, sheep and white-tailed deer from USA - A total of 253 isolates including 182 from pigs, 56 from sheep and 15 from white-tailed deer were analyzed. Of the

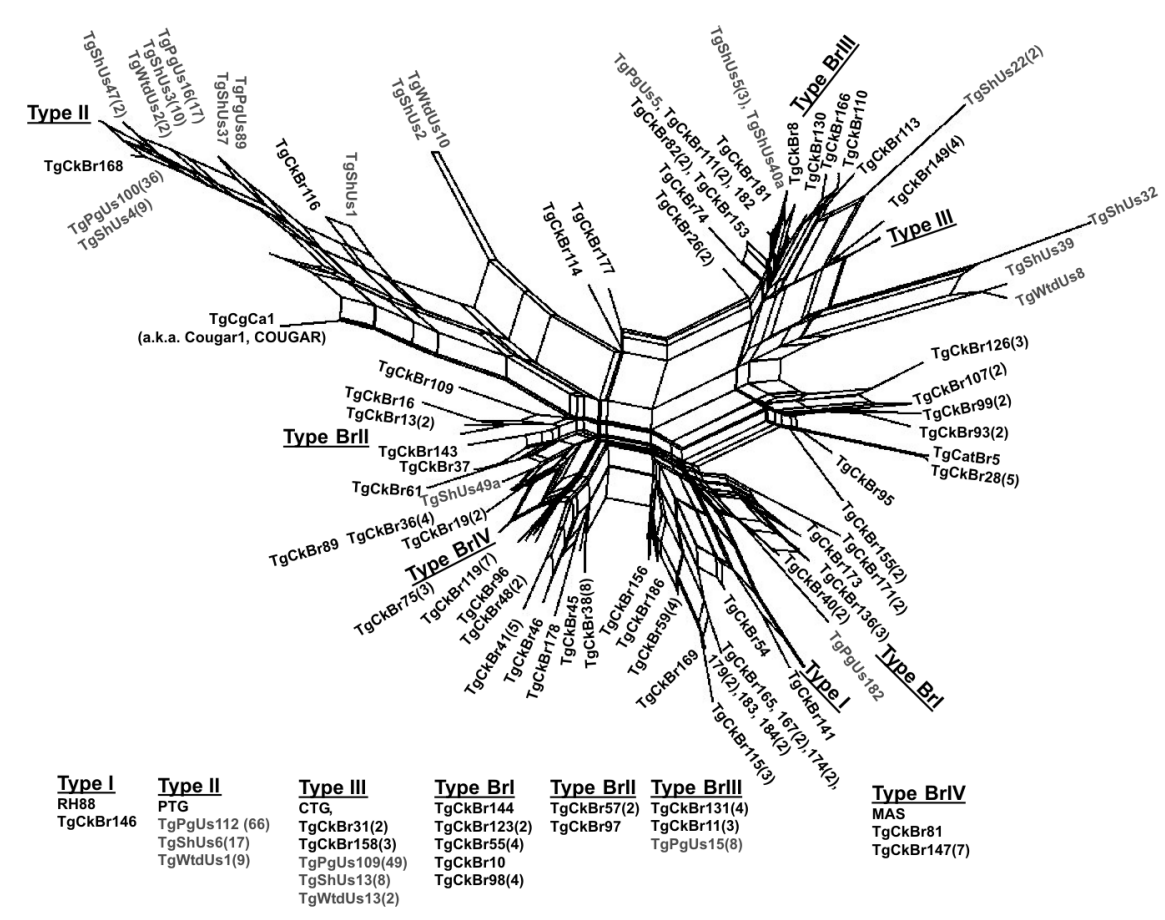

NeighborNet phylogenetic network of Toxoplasma gondii isolates. Isolates from animals in the USA are in red letters. RH88, PTG, CTG, $\mathrm{TgCgCa1}$ (a.k.a. Cougarl, COUGAR), MAS and TgCatBr5 are used as reference strains. Numeric number in parenthesis following each isolate's identification number is the number of strains with the identical genotype from the same states. 


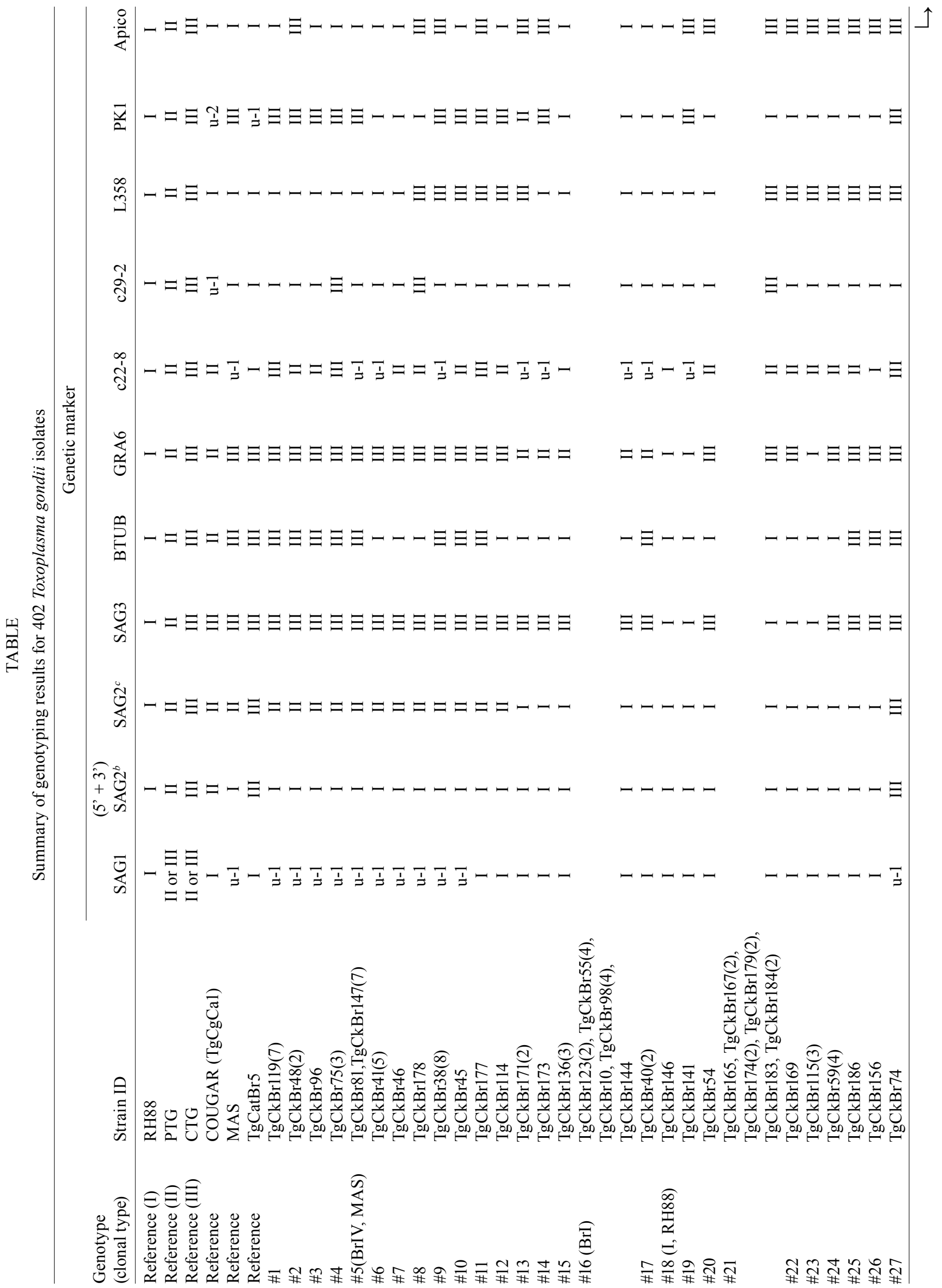


$\therefore \mid$ $\mid \uparrow$ 遂

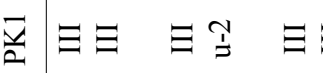

$\Xi \Xi \quad \Xi \Xi \quad \Xi \Xi$

$\Xi \Xi \Xi \Xi \Xi \Xi ー$

$-\Xi \quad \Xi \Xi \quad \Xi$

ヨョヨーーヨーーヨー

政

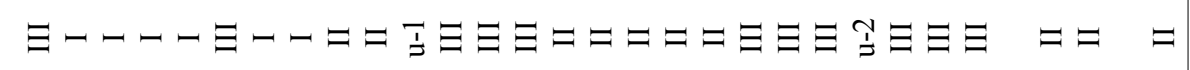

$\underset{\mathrm{U}}{\infty} \Xi \Xi \quad \Xi ョ \quad \Xi \Xi$

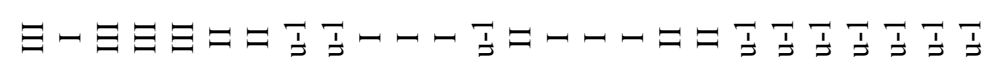

$\because ニ \quad ニ$

$\Xi \Xi \quad \Xi \Xi \quad \Xi \Xi$

$\Xi \Xi$

$\stackrel{n}{\underline{1}}$

$\Xi ー \quad \Xi \Xi \quad \Xi \Xi$

$\Xi \Xi \Xi \Xi \Xi$

$\Xi \Xi \quad \Xi \Xi \quad \Xi \Xi$

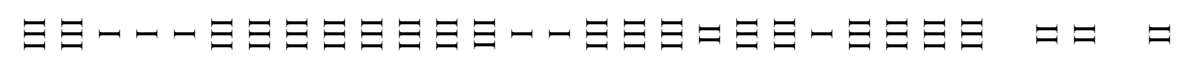

$\Xi \Xi \quad \Xi \Xi \quad \Xi \Xi$

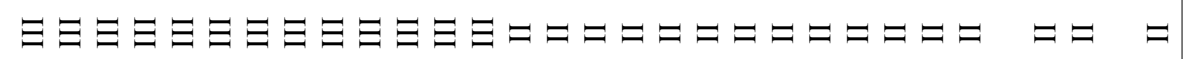

केषี

$\Xi \Xi \quad \Xi \Xi \quad \Xi \Xi$

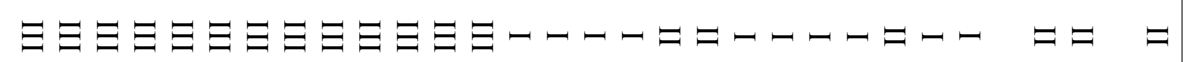

$\Xi \Xi$

岕

它

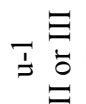

$\Xi \Xi$

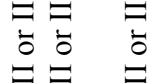

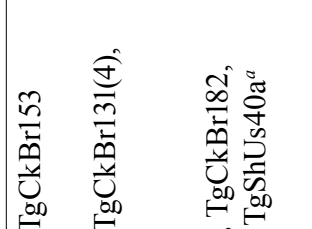

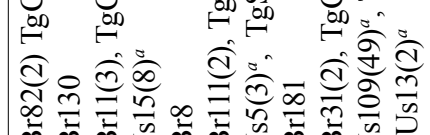

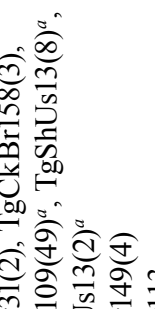




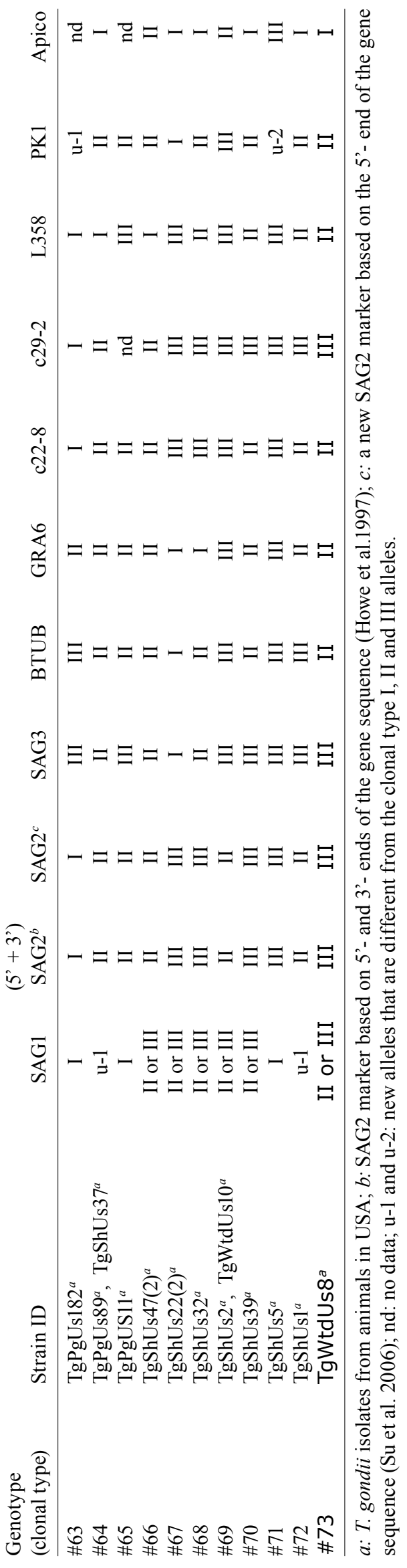

original 53 samples from sheep, four had mixed genotypes and three of the four are separated and indicated by "a" or "b", following the isolate designation. The one mixed genotype that could not be separated was not included for analysis.

Of the 253 isolates from animals, 18 genotypes were identified (Table, Figure). Ten of these genotypes have two or more isolates whereas eight genotypes only have one isolate each. Genotype \#60 and \#61 only differs at the Apico locus, and these groups were recognized as the type II lineage in the literatures due to the low resolution markers was used. Here, the collective type II is the predominant genotype and account for 54\% (137/253) of the samples originated from the USA. Genotype \#34 is the type III lineage, it accounts for $23 \%$ (59/253) of the samples and is the second major genotypes. Genotype \#62 accounts for $11 \%$ (29/253) of the isolates, it differs from \#61 only at the locus L358 and is closely related to the type II strains. The above three genotypes account for $89 \%$ (225 of 253) of the isolates from animals in the USA.

\section{DISCUSSION}

Isolates from Brazil - Three observations from these studies using a large number of unselected $T$. gondii isolates from a single host are noteworthy. First, there is absence of type II. Second, type I was rare. Third, most strains were not clonal, suggesting recombination and efficient transmission by oocysts. It is most likely, that humans and other animals in Brazil are exposed to oocysts of these isolates because soil contamination; isolates in chickens are an indicator of soil contamination.

Isolates from USA - Our studies indicate a higher genetic diversity than previously recognized. Pig is considered the main meat source of infection for humans in the USA, because the parasite has not been isolated from beef or chicken sold in the retail meat (Dubey et al. 2005). It is of interest all of the eight isolates in genotype $\# 30$ were from sows. Although the origin of each sow is unknown, they were unlikely to be from one farm because hundreds of sows were killed and processed each day at this abattoir and six of the seven isolates were from sows killed on different days. Why TgPgUs15 (formerly P89) type isolate was not found among isolates from market pigs is puzzling. Whether management or interaction with wildlife was a factor is a possibility. The sows might have been raised outdoors and would have had more interaction with other animals.

Overall, genetic diversity of the animal isolates from the USA is much lower than that of the chicken isolates from Brazil. In chicken isolates from Brazil, 58 genotypes were identified in $149 \mathrm{~T}$. gondii isolates, whereas only 18 genotypes were identified in 253 isolates from USA. The genotype with the highest frequency in Brazil samples is \#16 (type BrI), which only account for $8 \%$ (12 of 149) of the isolates, whereas 54\% (137 of 253) of the isolates from USA belong to the type II lineage, supporting the idea that $T$. gondii has a clonal population structure in North America but an epidemic population in Brazil (Howe \& Sibley 1995, Pena et al. 2008). Phylogenetic network analysis indicates that there are 
geographical structures between the two T. gondii populations in Brazil and USA (Figure). Most isolates from USA are clustered into the clonal type II and III lineages, whereas isolates from Brazil are scattered and can be loosely subdivided into three clusters including type III/type BrIII, type I/type BrI and type BrII/type BrIV. The different population structures of $T$. gondii between Brazil and USA may be shaped by different transmission routes, which is of interest to understand the epidemiology of the parasite.

\section{REFERENCES}

Dubey JP 2007. The history and life cycle of Toxoplasma gondii. In LM Weiss and K Kim, Toxoplasma gondii. The model apicomplexan: perspectives and methods. Academic Press, New York, p. 1-17.

Dubey JP, Graham DH, Blackston CR, Lehmann T, Gennari SM, Ragozo AMA, Nishi SM, Shen SK, Kwok OCH, Hill DE, Thulliez P 2002. Biological and genetic characterisation of Toxoplasma gondii isolates from chickens (Gallus domesticus) from São Paulo, Brazil: unexpected findings. Int J Parasitol 32: 99-105.

Dubey JP, Hill DE, Jones JL, Hightower AW, Kirkland E, Roberts JM, Marcet PL, Lehmann T, Vianna MCB, Miska K, Sreekumar C, Kwok OCH, Shen SK, Gamble HR 2005. Prevalence of viable Toxoplasma gondii in beef, chicken and pork from retail meat stores in the United States: risk assessment to consumers. J Parasitol 91: 1082-1093.

Dubey JP, Hill DE, Sundar N, Velmurugan GV, Bandini LA, Kwok OCH, Pierce V, Kelly K, Dulin M, Thulliez P, Iwueke C, Su C 2008a. Endemic toxoplasmosis in pigs on a farm in Maryland: isolation and genetic characterization of Toxoplasma gondii. J Parasitol 94: 36-41.

Dubey JP, Jones JL 2008. Toxoplasma gondii infection in humans and animals in the United States. Int J Parasitol 38: 1257-1278.

Dubey JP, Patitucci AN, Su C, Sundar N, Kwok OCH, Shen SK 2006. Characterization of Toxoplasma gondii isolates in free-range chickens from Chile, South America. Vet Parasitol 140: 76-82.

Dubey JP, Rollor EA, Smith K, Kwok OCH, Thulliez P 1997. Low seroprevalence of Toxoplasma gondii in feral pigs from a remote island lacking cats. J Parasitol 83: 839-841.
Dubey JP, Sundar N, Velmurugan GV, Bandini LA, Kwok OCH, Majumdar D, Su C 2008b. High prevalence and abundant atypical genotypes of Toxoplasma gondii isolated from lambs destined for human consumption in the USA. Int J Parasitol 38: 999-1006.

Dubey JP, Velmurugan GV, Chockalingam A, Pena HFJ, de Oliveira LN, Leifer CA, Gennari SM, Oliveira LMGB, Su C 2008c. Genetic diversity of Toxoplasma gondii isolates from chickens from Brazil. Vet Parasitol 157: 299-305.

Dubey JP, Velmurugan GV, Ulrich V, Gill J, Carstensen M, Sundar N, Kwok OCH, Thulliez P, Majumdar D, Su C 2008d. Transplacental toxoplasmosis in naturally-infected white-tailed deer: isolation and genetic characterization of Toxoplasma gondii from foetuses of different gestational ages. Int J Parasitol 38: 1057-1063.

Howe DK, Honoré S, Derouin F, Sibley LD 1997. Determination of genotypes of Toxoplasma gondii strains isolated from patients with toxoplasmosis. J Clin Microbiol 35: 1411-1414.

Howe DK, Sibley LD 1995. Toxoplasma gondii comprises three clonal lineages: correlation of parasite genotype with human disease. J Infect Dis 172: 1561-1566.

Lehmann T, Marcet PL, Graham DH, Dahl ER, Dubey JP 2006. Globalization and the population structure of Toxoplasma gondii. Proc Natl Acad Sci 103: 11423-11428.

Mateus-Pinilla NE, Dubey JP, Choromanski L, Weigel RM 1999. A field trial of the effectiveness of of a feline Toxoplasma gondii vaccine in reducing $T$. gondii exposure for swine. $J$ Parasitol 85: $855-860$.

Munday BL 1972. Serological evidence of Toxoplasma infection in isolated groups of sheep. Res Vet Sci 13: 100-102.

Pena HFJ, Gennari SM, Dubey JP, Su C 2008. Population structure and mouse-virulence of Toxoplasma gondii in Brazil. Int J Parasitol 38: 561-569.

Su C, Zhang X, Dubey JP 2006. Genotyping of Toxoplasma gondii by multilocus PCR-RFLP markers: a high resolution and simple method for identification of parasites. Int J Parasitol 36: 841-848.

Velmurugan GV, Su C, Dubey JP 2009. Isolate designation and characterization of Toxoplasma gondii isolates from pigs in the United States. J Parasitol 94: 95-99.

Wallace GD, Marshall L, Marshall M 1972. Cats, rats and toxoplasmosis on a small Pacific island. Am J Epidemiol 95: 475-482. 